CIC. Cuadernos de Información y Comunicación ISSN: 1135-7791

http://dx.doi.org/10.5209/CIYC.5209

\title{
Estilos de producción en el campo de las ciencias sociales en Argentina
}

\author{
Juan Ignacio Piovani ${ }^{1}$
}

Recibido: 15 de mayo de 2018 / Aceptado: 6 de julio de 2018

Resumen. Muchos estudios han analizado el desarrollo de un lenguaje y estilo de escritura "universales" en la ciencia. También ha sido objeto de interés el grado en que este estilo ha permeado diversos contextos académicos nacionales. Argentina es un caso interesante porque en tiempos recientes experimentó un incremento del financiamiento científico que permitió la expansión de la investigación, la contratación de cientos de nuevos investigadores de tiempo completo y la consolidación de un sistema de becas de doctorado. A su vez, esto condujo a un aumento notable de las publicaciones. Sin embargo, a pesar de que todo ello ocurrió en el marco de un proceso de creciente profesionalización (que implica mayores niveles de alineamiento con los estándares científicos internacionales), estudios actuales muestran que en Argentina prevalecen dos modelos antagónicos en las ciencias sociales: uno que replica las prácticas y estándares internacionales vigentes y otro, de carácter más bien endógeno, con sus propias lógicas de producción, evaluación y circulación del conocimiento. Con el fin de examinar el impacto de los estándares internacionales en las ciencias sociales argentinas, en este artículo se analizan los estilos de producción académica. Esto implica dar cuenta de tres dimensiones íntimamente relacionadas: modelos y procesos de investigación (orientaciones teóricas, métodos, técnicas, etc.); formatos de escritura (estructura y organización de textos académicos) y lógicas de publicación (tipos y perfiles de revistas y otros soportes). El análisis se basa en una amplia muestra de artículos basados en investigaciones empíricas que fueron seleccionados por pares con el fin de realizar una revisión de la literatura especializada sobre seis temas clave de las ciencias sociales. Pero los artículos también fueron examinados para producir una base de datos con múltiples variables relacionadas con las tres dimensiones apenas mencionadas. Los resultados muestran que más allá del reciente proceso de profesionalización, en las ciencias sociales de Argentina aún prevalece un modelo "heterodoxo" de producción académica.

Palabras clave: Argentina, ciencias sociales, estilos de producción.

\section{[en] Styles of production in the field of social sciences in Argentina}

Abstract. Several studies have analyzed the development of a "universal" scientific language and
writing style. The extent to which this style has permeated diverse national academic contexts has
also been a matter of interest. Argentina is an appealing case for analyzing this phenomenon because
in recent years there has been a robust increase in public funding, which gave way to the expansion of
research, the recruitment of hundreds of new full-time researchers, and the consolidation of fellowships
for PhD students. All these, in turn, resulted in a remarkable increase in publications. However, even
though these processes have occurred in the midst of professionalization (which implies higher levels
of attachment to international academic standards), recent studies have shown that in Argentina still
prevail two contesting models within the social sciences: one that conforms to international standards
and practices, and another one of a more endogenous nature, with its own logic for knowledge
production, evaluation and circulation. In order to examine the impact of the international standards
in Argentinian social sciences, in this paper I analyze the styles of academic production. This implies

1 CONICET / UNLP. 
the study of three closely related dimensions: research processes \& models (theoretical foundations, methods, techniques, etc.); writing formats (structure and organization of academic texts); publication logic (types and profiles of journals where these texts are published). The analysis is based on a large sample of articles (grounded on empirical research) that were selected by peers in order to carry out a comprehensive review of Argentinian literature with regard to six key themes of the social sciences. But the articles were also examined meticulously to produce a dataset with several variables related to the three above-mentioned dimensions. The results show that regardless of the recent process of professionalization, a "heterodox model" of academic production is still largely pervasive within Argentinian social sciences.

Keywords: Argentina, social sciences, styles of production

Sumario. Introducción. Métodos. Resultados. Discusión y conclusiones. Referencias. Anexo.

Cómo citar: Piovani, J. I. (2018). Estilos de producción en el campo de las ciencias sociales en Argentina, en CIC. Cuadernos de Información y Comunicación 23, 125-141.

\section{Introducción}

En el año 2012, a instancias del Consejo de Decanos de Facultades de Ciencias Sociales y Humanas de la Argentina (CODESOC), y con financiamiento del Ministerio de Ciencia, Tecnología e Innovación Productiva (MINCYT) y de la Secretaría de Políticas Universitarias (SPU), comenzó a ejecutarse el Programa de investigación sobre la sociedad argentina contemporánea (PISAC).

Los orígenes del PISAC se remontan al año 2009, cuando el Ministro aceptó financiar un gran programa nacional de ciencias sociales luego de haber puesto en cuestión, en una entrevista periodística, su carácter científico: en efecto, adujo que ellas requerían una revolución metodológica para convertirse en verdaderas ciencias y comparó su conocimiento con la teología.

La amplia participación de la comunidad académica local en el diseño del programa PISAC fue clave para elaborar un diagnóstico del estado de situación de las ciencias sociales en el país, que puso en evidencia realidades contradictorias. Por un lado, se destacó la reinstitucionalización de la investigación y de la educación superior a partir de la recuperación democrática de la década de 1980, así como la gran expansión y diversificación temática de la investigación social, en especial en las últimas dos décadas. Por otro lado, se reconoció el carácter fragmentado de la producción científica, la escasa circulación de los resultados de las investigaciones y su bajo impacto en el diseño de políticas públicas, entre otras cosas.

A partir de este diagnóstico se definieron tres líneas de trabajo. La primera apuntó a relevar las investigaciones recientes en torno de seis núcleos temáticos clave -1) Estructura social; 2) Condiciones de vida; 3) Estado, gobierno y administración pública; 4) Ciudadanía, movilización y conflicto social; 5) Diversidad sociocultural; 6) Consumos culturales- con el fin de sistematizar los conocimientos disponibles y visibilizar los resultados de las investigaciones a través de libros que se estructuraron como "estados de la cuestión".

Por otra parte, resultó evidente que, más allá de investigar sobre temas específicos, era oportuno indagar sobre el sistema nacional de ciencias sociales. Esto constituyó el núcleo de una segunda línea de trabajo, cuyas preguntas centrales fueron: ¿cómo está estructurado el sistema de investigación y de educación superior en el campo de 
las ciencias sociales en la Argentina actual? ¿Qué tipo de asimetrías interinstitucionales e interregionales presenta? ¿Cómo se han conformado las agendas de investigación y qué problemas han sido más estudiados? ¿Cómo son los perfiles y las trayectorias académicas de las/os docentes e investigadoras/es en actividad? ¿Cuáles han sido las orientaciones teóricas y las perspectivas metodológicas predominantes? ¿Cómo se caracteriza la producción académica de las ciencias sociales, por qué medios se publica y en qué ámbitos circula? (Piovani, 2015a)

Finalmente, una tercera línea de indagación tuvo como objetivo relevar la heterogeneidad estructural de la sociedad argentina a partir del análisis de datos primarios. Para ello se diseñaron tres encuestas nacionales: sobre la estructura social y las condiciones de vida; sobre las relaciones sociales; y sobre los valores, las actitudes y las representaciones sociales (Piovani, 2017).

Este artículo se enmarca en la segunda línea de investigación del PISAC, aunque los datos empíricos en los que se basa resultaron del análisis del corpus de publicaciones que se utilizó para construir los estados de la cuestión sobre los núcleos temáticos apenas mencionados (primera línea). El objetivo principal de este trabajo es describir los estilos de producción de las ciencias sociales en Argentina. Esto implica analizar de tres dimensiones íntimamente relacionadas: modelos y procesos de investigación (orientaciones teóricas, estrategias metodológicas, técnicas utilizadas, etc.); formatos de escritura (estructura y organización de los textos académicos) y lógicas de publicación (tipos y perfiles de revistas y otros soportes).

En un sentido amplio, la preocupación en la que se inscribe este trabajo se relaciona con el creciente peso que, a nivel mundial -y muy especialmente en los Estados Unidos-, ha adquirido lo que Burawoy (2005) define como professional socio$\log y$, que se caracteriza por la producción de un conocimiento eminentemente instrumental, de tipo teórico/empírico, a partir de programas de investigación que se desarrollan en el ámbito académico y cuya expresión tangible es el paper. De acuerdo con Burawoy, la legitimidad de este conocimiento está garantizada por las normas científicas y sus mecanismos de control se basan en la evaluación por pares.

Desde el punto de vista de su desarrollo histórico y de su progresiva consolidación como modelo hegemónico del trabajo académico institucionalizado, la professional sociology -que en mayor o menor medida puede extenderse al conjunto de las ciencias sociales empíricas- no puede desvincularse de lo que Beigel y Salatino (2015: 12) denominan Sistema Académico Mundial, caracterizado por "una estructura desigual de producción y circulación de conocimiento, cuyo centro dominante es el llamado circuito 'mainstream' o de corriente principal." De acuerdo con estos autores, y como parte de este sistema mundial, a partir de mediados del siglo XX comenzó a priorizarse a los papers por sobre otras formas de producción y diseminación del conocimiento, un proceso que fue más tardío en las ciencias sociales pero que terminó por instalarse también en ellas durante la década de 1990.

El Sistema Académico Mundial tiene como uno de sus principales pilares un sistema de publicaciones que progresivamente ha establecido un lenguaje y un estilo de escritura "universal", y que está compuesto por revistas líderes indizadas en bases de datos internacionales de carácter comercial (Beigel, 2017a), con sesgo en favor de las publicaciones en lengua inglesa y con escasa representatividad de revistas de ciencias sociales y humanas (Ortiz, 2009)

Este estilo de escritura "universal" se materializa, en buena medida, en el modelo IMRAD (Introduction, Methods, Results, Discussion) y en el uso del inglés como 
lingua franca de la ciencia. Según Day (1989), este formato fue consagrado como modelo estándar del artículo científico en 1972, luego de la publicación del American National Standard for the preparation of scientific papers for written or oral presentation (ANSI), que en los años siguientes fue adoptado por decenas de organizaciones científicas y de revistas especializadas.

Esta afirmación sobre la consagración del modelo IMRAD a partir de la década de 1970 es consistente con los resultados de la investigación sobre artículos científicos del campo de las ciencias de la salud, publicados en 4 revistas líderes ${ }^{2}$ entre 1935 y 1985, llevada a cabo por Sollaci y Pereira (2004). Ellos mostraron que el uso del formato IMRAD, en sentido amplio, comenzó a registrarse en la década de 1940, pero recién se generalizó en la década de 1970, cuando alcanzó al 80\% de los artículos publicados, y se convirtió en el único patrón adoptado en artículos originales en la década siguiente.

Si bien este formato ha tenido mayor impacto en las ciencias naturales y, en particular, en las ciencias biomédicas, también se ha difundido en las ciencias sociales, e incluso ha sido adoptado por algunas revistas profesionalizadas de las artes y las humanidades (Day, 1989). La Academy of Social Sciences and Humanities (ASSH) señala que aunque el formato IMRAD es más común en física y biología, también se utiliza ampliamente en las ciencias sociales y de la conducta, especialmente en la Psicología, dado que la American Psychological Association (APA), en su manual de estilo, propone la estructura IMRAD para los encabezados de las secciones de un artículo académico. ${ }^{3}$ No obstante, como señala la ASSH, la investigación social y humanística suele usar un formato similar al IMRAD, en el que es habitual la inclusión de una sección dedicada al análisis de antecedentes / revisión de literatura / estado de la cuestión. Al respecto, cabe señalar que antes de su estandarización durante la década de 1970, esta sección también era corriente en los papers de las ciencias naturales bajo el título de "Trabajo Previo" (Brain, 1965).

En la expansión del modelo IMRAD ha sido importante la influencia de otras disciplinas, como se acaba de ver para el caso de las ciencias sociales, y las recomendaciones de los editores de las revistas científicas (Sollaci y Pereira, 2004), así como las políticas públicas e institucionales en materia de ciencia y técnica que, en no pocos casos, han orientado las estrategias de publicación de los académicos hacia el sistema mainstream, de mayor prestigio y, supuestamente, también de mayor impacto. Tampoco puede desconocerse el peso de la ya ubicua práctica de evaluación de la actividad científica, que tiene un claro efecto performativo (Piovani, 2015b) modelando las decisiones de los investigadores sobre estilos escriturales y, más fundamentalmente, sobre estrategias de publicación (aunque posiblemente también ejerza influencia sobre la definición de objetos de investigación, teorías, enfoques y métodos), tal como sugiere Hanafi (2011) en su análisis de la producción de cientistas sociales del mundo árabe que se consagran en el circuito mainstream.

En el marco de estas dinámicas globales resulta relevante analizar los estilos de producción de las ciencias sociales en contextos periféricos, marcados por lo que Alatas (2003) denomina "dependencia académica." Si bien ha sido habitual asumir que ellos se encuentran fuertemente expuestos a la influencias de los modelos extranjeros dominantes, Beigel (2017a) sostiene que la dependencia académica en realidad

British Medical Journal, JAMA, The Lancet y New England Journal of Medicine.

Véase https://asshacademy.wordpress.com/2011/07/17/the-imrad-research-paper-format/ 
"altera las culturas evaluativas nacionales y profundiza la heterogeneidad estructural", segmentado los circuitos de consagración académica.

El caso argentino es especialmente interesante porque se trata de un sistema científico que luego de la crisis de 2001/2, y hasta 2015, experimentó una enorme expansión. Inmediatamente después de la crisis la entonces Secretaría de Ciencia y Técnica comenzó la elaboración de un Plan Estratégico Nacional de Ciencia, Tecnología e Innovación que fijó como metas, entre otras, aumentar la inversión en I+D hasta el $1 \%$ del PIB y llevar el número de investigadores y tecnólogos al 3 por mil de la PEA (Unzué, 2015). En 2007 se creó el Ministerio de Ciencia, Tecnología e Innovación Productiva (MINCYT), lo que significó una jerarquización de la agenda científica dentro de la política pública y que, a la vez, permitió contar con espacios institucionales especializados para planificarla y ejecutarla.

En el marco de estas políticas de expansión, el Consejo Nacional de Investigaciones Científicas y Técnicas (CONICET), principal órgano argentino en materia científica, tuvo un notable incremento en su planta de investigadores de ciencias sociales y humanas — de 705 (en 2003) a 2.245 (en 2016) - y de becarios doctorales —de 493 (en 2003) a 2.896 (en 2016) —. Igualmente relevante fue la ampliación de la oferta de programas de formación doctoral, especialmente en las universidades públicas, y de subsidios para financiar proyectos de investigación. También se verificó una notable revitalización del mercado editorial vinculado con las ciencias sociales y del sistema de revistas especializadas, la mayoría de ellas editadas por instituciones científicas o de educación superior.

Esta expansión fue a la par de un creciente proceso de profesionalización de las prácticas académicas y científicas que, aunque con sus propias dinámicas, siguió algunos de los principales patrones internacionales. Entre otras cosas, se reforzó la institucionalización de las lógicas de evaluación -siguiendo en general el modelo de evaluación por pares- y, si bien de modo generalmente indirecto, se incentivó la publicación en revistas mainstream de prestigio, que en el sistema argentino pasaron a denominarse revistas "Tipo 1". En este sentido, "en el campo de las ciencias sociales se puede citar el trabajo de una comisión del Consejo Nacional de Investigaciones Científicas y Técnicas (CONICET), que se materializó en la Resolución 2249/14: "Bases para la Categorización de Publicaciones Periódicas en Ciencias Sociales y Humanidades" (Peirano, Freibrun y Sleiman, 2015: 11).

No obstante, algunas investigaciones recientes han puesto en evidencia que la difusión del modelo dominante de producción científica-centrado en el paper en lengua inglesa y publicado en revistas de corriente principal- presenta peculiaridades en las ciencias sociales en Argentina y otros contextos periféricos.

Beigel (2017a, 2017b), por ejemplo, señala la persistencia de dos modelos dentro de este campo: uno vinculado a los científicos integrados a los estilos de producción dominantes en el sistema académico mundial y otro representado por aquellos que tienen una agenda más endógena, que se restringen a circuitos locales nutridos de numerosas revistas sin indización, publicadas es español y con frecuencia solo en soporte impreso. De acuerdo con esta autora, la expansión del sistema científico de los últimos años profundizó la polarización entre estos dos modelos. Baranger y Niño (e/p), por su cuenta, han complejizado el análisis propuesto por Beigel a partir de la incorporación de otras variables y, como resultado de su investigación sobre la producción de cientistas sociales argentinos, han propuesto una tipología de 5 estilos de publicación, que van desde uno estándar (que se asemeja al perfil predominante 
de las ciencias naturales) hasta otros proto-estándar, localistas (en dos versiones) y universalista, que implica una orientación fuerte hacia las publicaciones internacionales, pero no en inglés sino en otros idiomas como francés, portugués, alemán e italiano.

Tendiendo este conjunto de problemas y análisis como trasfondo, en este artículo proponemos la descripción de un corpus de publicaciones recientes de las ciencias sociales argentinas, tal como ya se ha señalado, con énfasis en los artículos de revista que se basan en investigaciones empíricas, bajo el supuesto de que ellos representan más cabalmente el modelo de producción de conocimiento propiciado en el marco del Sistema Académico Mundial. En particular, focalizamos la estructura formal de los artículos, algunos aspectos metodológicos y técnicos de las investigaciones que les dieron origen, sus citas y referencias bibliográficas, las características de las revistas en las que fueron publicados $\mathrm{y}$, aunque de manera relativamente acotada, su impacto.

\section{Métodos}

Esta investigación se basa en el análisis de un corpus de 421 publicaciones de ciencias sociales cuyo marco temporal de producción coincide con el de la expansión del sistema científico argentino luego de la crisis de 2001/2002.

El corpus se conformó con textos seleccionados por los investigadores que llevaron adelante la elaboración de los estados de la cuestión sobre cinco de los seis núcleos temáticos señalados en la introducción de este artículo (quedó excluido el de condiciones de vida). Si bien no se trata de una muestra aleatoria de la producción de las ciencias sociales durante el periodo analizado, el corpus es relevante porque incluye las publicaciones que los pares de diversas instituciones y regiones del país consideraron pertinentes para dar cuenta de manera integral de la investigación social argentina sobre los temas citados.

Para constituir el corpus se llevó a cabo un muestreo intencional organizado a partir de un conjunto de matrices:

1. Matriz de TEMAS / DIMENSIONES / PERSPECTIVAS (teóricas y metodológicas) / PROBLEMAS / HIPOTESIS (en relación con cada uno de los núcleos temáticos)

2. Matriz de REGIONES / INSTITUCIONES / PERFILES DE AUTORES (en cuanto a las regiones se tuvo en cuenta un doble aspecto: a) producida en $\mathrm{x}$ región y b) acerca de $\mathrm{x}$ región.

3. Matriz de TIPO DE PUBLICACIÓN (libro / capitulo / articulo / ponencia) / TIPO DE TRABAJO (ensayo / investigación teórica / investigación empírica, etc.).

4. Matriz de DIMENSION TEMPORAL (delimitación temporal como OBJETO DE ANÁLISIS y como FECHA DE PUBLICACIÓN).

Cada matriz quedó definida por una cantidad variable de celdas. La posterior yuxtaposición de las diferentes matrices multiplicó la cantidad de celdas, con una complejidad creciente que permitió reconstruir la heterogeneidad del campo de producción académica sobre la base de las dimensiones consideradas en el conjunto de 
las matrices. Obviamente, no se pretendió cubrir hasta el más mínimo detalle resultante de las matrices combinadas, sino contar con un marco de referencia que permitiera cumplir con los objetivos de exhaustividad (temática, regional, etc.) en la cobertura de la producción académica. En definitiva, se buscó evitar deliberadamente que el análisis se concentrara en un único tipo de producción, en pocos autores de una misma región o institución, acerca de un mismo tema (o dimensión) o sobre perspectivas muy afines entre sí (Piovani, 2015a).

Para hacer operativa la búsqueda y posterior selección de publicaciones se recurrió a una serie de estrategias y fuentes complementarias:

- Búsqueda a través de bases de datos o repositorios, usando filtros, palabras clave y descriptores.

- Búsqueda e identificación a través de colegios invisibles y referencias de informantes clave (expertos en la materia).

- Búsqueda a través de listados de revistas $\rightarrow$ índices de artículos / índices de autores.

- Búsqueda a través de listados de editoriales $\rightarrow$ colecciones de libros $\rightarrow$ títulos de libros $\rightarrow$ índices de libros.

- Búsqueda a través de listados de congresos $\rightarrow$ listados de mesas temáticas $\rightarrow$ índices de ponencias / índices de autores.

- Búsqueda basada en la exploración de los listados de centros e institutos de investigación $\rightarrow$ equipos $\rightarrow$ líneas $\rightarrow$ proyectos $\rightarrow$ producciones enmarcadas en los proyectos.

- Búsqueda a partir de una estrategia "bola de nieve" (Valles, 1997) tomando las referencias bibliográficas de otros trabajos que ya habían sido identificados y seleccionados.

Como ya se ha señalado, las publicaciones incluidas en el corpus fueron seleccionadas con la finalidad principal de elaborar estados de la cuestión. Pero también se las relevó a los efectos de producir insumos -datos primarios- para esta investigación sobre estilos de producción. En este sentido, se aplicó de manera transversal un instrumento diseñado como marco de codificación (véase Anexo) que incluyó 28 ítems generales y otros específicos para cada tipo de publicación ${ }^{4}$ y de trabajo. ${ }^{5}$

El marco de codificación abarcó un amplio abanico de cuestiones relacionadas con los textos (estructura, bibliografía, impacto, etc.), las investigaciones en las que se basan (financiamiento, colaboración nacional o internacional, fundamentos teóricos, decisiones metodológicas, etc.), los autores (cantidad, afiliación institucional, perfil, etc.) y los soportes de publicación (así como sobre las características de las revistas, en el caso de los artículos).

\footnotetext{
Libro, capitulo de libro, artículo de revista científica, ponencia presentada en congreso.

Ensayo (trabajo de argumentación / reflexión /opinión personal sobre un tema /problema, más allá de que contenga referencias teóricas y/o empíricas); Estudio propositivo - normativo (trabajo cuyo objetivo es proponer una intervención social determinada, una política social específica o una guía de acción o práctica, más allá de que contenga referencias teóricas y/o empíricas); Investigación teórica (trabajo que se centra en la discusión sistemática de una perspectiva o concepto, o en la construcción conceptual sobre un tema / problema, aun conteniendo referencias a casos empíricos); Estado de la cuestión - revisión de literatura (trabajo que da cuenta, sistemáticamente, del conjunto de publicaciones sobre un tema / problema); Investigación empírica (trabajo que se basa en la producción y análisis de datos primarios producidos a través de cualquier tipo de metodología científica, o en el análisis sistemático de datos secundarios, más allá de que contenga referencias teóricas)
} 
El relevamiento dio lugar a la elaboración de una base de datos de 97 variables, a las que se agregaron otras 15 construidas a partir del procesamiento de los datos. $\mathrm{La}$ base se analizó estadísticamente utilizando el paquete SPSS. La mayoría de los análisis fueron de tipo monovariado - distribuciones de frecuencias- o bivariado -basado en tablas de contingencia-.

\section{Resultados}

En primer lugar se presenta un panorama general del corpus analizado, sin diferenciar por tipo de publicación o de trabajo. Esto resulta relevante porque, como se verá a continuación, el corpus no solo incluye artículos de revistas o trabajos de carácter empírico, sino que contiene otros tipos de formatos y estilos de producción académica. Tal como se observa en el gráfico 1, el 46,5\% son artículos de revistas científicas, el $21,9 \%$ son capítulos de libros, el $20,2 \%$ son ponencias presentadas en congresos y el $11,4 \%$ son libros completos.

El $65,8 \%$ de las publicaciones es de autoría individual; $21,9 \%$ es de dos autores; $7,6 \%$ de tres autores y el restante $4,7 \%$ de 4 o más autores. En el $86 \%$ de los casos se consigna la afiliación institucional, y el $40,6 \%$ se produjo en el marco de un programa o proyecto de investigación acreditado, o a partir de la investigación para una tesis de posgrado. Pero solo en el $27,1 \%$ de los textos se declara haber recibido un subsidio o algún tipo de financiamiento (ya sea para la investigación o para su publicación). Entre las fuentes de financiamiento destacan, en primer lugar, las universidades nacionales. Aunque en menor proporción, también son importantes los subsidios de la Agencia Nacional de Promoción Científica y Tecnológica (a través del

Gráfico 1. Tipos de publicaciones.

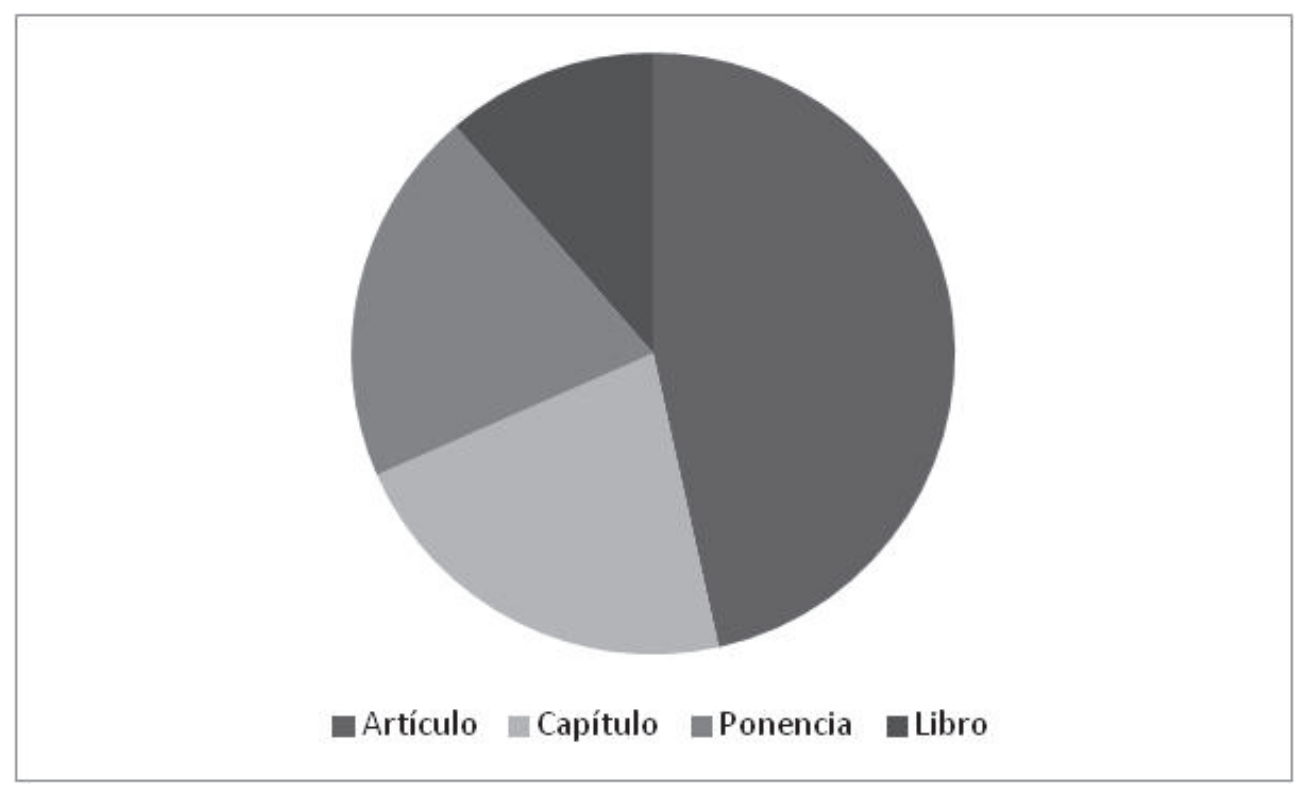

Fuente: elaboración propia a partir de la base EC-PISAC. 
FONCYT) y del Consejo Nacional de Investigaciones Científicas y Técnicas (CONICET). Según la información recabada, el 17,8\% de las publicaciones analizadas ha sido fruto del trabajo colaborativo (entre grupos de investigación / instituciones / países), con porcentajes casi equivalentes en los niveles de colaboración local, nacional e internacional.

En cuanto a los tipos de trabajos, teniendo en cuenta las definiciones utilizadas en esta investigación (véase nota 4), se constata que $17,1 \%$ son ensayos; $3,8 \%$ estudios normativos-propositivos; $12,1 \%$ investigaciones teóricas; $5,7 \%$ estados de la cuestión o revisiones de literatura; $60,3 \%$ investigaciones empíricas y $1 \%$ estudios de otro tipo.

La distribución regional de la producción incluida en el corpus es la siguiente:

- Capital Federal: 36,3\%

- Metropolitana (Gran Buenos Aires, excluida la Capital Federal): 11,7\%

- Pampeana (resto de la prov. de Buenos Aires y La Pampa): 9\%

- Centro (Córdoba y Santa Fe): 12,1\%

- Cuyo (Mendoza, San Juan y San Luis): 7,2\%

- Noreste-Litoral (Entre Ríos, Corrientes, Chaco, Misiones y Formosa): 8,6\%

- Noroeste (Jujuy, Salta, Tucumán, Santiago del Estero, La Rioja y Catamarca): $7,1 \%$

- Patagonia (Rio Negro, Chubut, Neuquén, Santa Cruz y Tierra del Fuego): $6,4 \%$

- Otra (publicaciones de argentinos en el exterior): $1,4 \%$

De los núcleos temáticos considerados el aporte más bajo al corpus $(13,5 \%$ del total) fue el del núcleo sobre diversidad sociocultural, y el más alto $(24,9 \%)$ fue el del núcleo sobre consumos culturales. Los restantes contribuyeron con 19,7\% (Estado, gobierno y administración pública), 20,2\% (ciudadanía, movilización y conflicto social) y $21,6 \%$ (estructura social).

Para avanzar con el análisis de las publicaciones nos concentramos a continuación exclusivamente en los artículos de revistas basados en investigaciones empíricas. Desde el punto de vista estructural, se observa que el 79,8\% de ellos cuenta con lo que podríamos definir como "marco teórico o conceptual", a la vez que el 57\% presenta un estado de la cuestión o revisión de literatura y el 36,8\% contiene una sección o apartado dedicado a la metodología. En línea con esto último, el 43,9\% presenta alguna hipótesis de manera explícita.

Si se profundiza en el análisis metodológico de las investigaciones en las que se basan estos artículos se constata que $45,6 \%$ de ellas fueron de carácter cualitativo, $26,3 \%$ cuantitativo y $28,1 \%$ recurrieron a métodos mixtos o diversas formas de triangulación metodológica. En el 31,2\% de los casos se utilizaron datos primarios, en $29,3 \%$ datos secundarios y en $39,5 \%$ ambos tipos de datos. Apenas en el $14 \%$ de los artículos se reporta haber utilizado algún software para el análisis de los datos.

Por otra parte, el ámbito espacial de las investigaciones fue, mayoritariamente, de nivel local (43\%), seguido por los estudios de nivel nacional (29\%) -basados en este caso en datos secundarios- y regional (19,3\%). Los análisis latinoamericanos $(4,4 \%)$ e internacionales $(0,9 \%)$ resultaron más bien marginales desde el punto de vista de su peso en el conjunto de artículos seleccionados. 
Ya se dijo con anterioridad que solo el 36,8\% contiene un apartado en el que se explicitan las estrategias metodológicas. Pero más allá de esto, también se indagó acerca de las decisiones específicas en relación con el muestreo, la recolección de datos y su análisis. Los resultados presentados en la tabla 1 muestran que, en general, es mucho mayor la propensión a consignar de manera explícita las técnicas de recolección de datos utilizadas: éstas se declaran en más del $83 \%$ de los casos. En el otro extremo, en una mayoría de los artículos (más del 63\%) no se encuentran alusiones concretas a las técnicas de muestreo empleadas. Asimismo, se pudo establecer que, tendencialmente, los artículos basados en investigaciones con métodos cuantitativos o mixtos explicitan en mayor medida sus decisiones de muestreo y de análisis. En relación con las decisiones de recolección, en cambio, no se encontró una asociación estadística significativa entre la propensión a explicitarlas y el tipo de estrategia metodológica.

Tabla 1. Técnicas de muestreo, recolección y análisis de datos.

\begin{tabular}{|l|c|l|l|l|l|}
\hline \multicolumn{2}{|c|}{ Técnicas de Muestreo } & \multicolumn{2}{c|}{ Técnicas de recolección } & \multicolumn{2}{c|}{ Técnicas de análisis } \\
\hline No se consignan & $63,2 \%$ & No se consignan & $16,7 \%$ & No se consignan & $33,3 \%$ \\
\hline Muestreo probabilistico & $7,9 \%$ & Encuesta & $20,2 \%$ & Análisis estadístico & $28,1 \%$ \\
\hline Muestreo intencional & $14,0 \%$ & Entrevista & $33,3 \%$ & Análisis de contenido & $17,5 \%$ \\
\hline Otras & $14,9 \%$ & Observación documental & $14,0 \%$ & Otras & $21,1 \%$ \\
\cline { 3 - 5 } & & Otras & $15,8 \%$ & & \\
\cline { 3 - 4 } & & & & &
\end{tabular}

Fuente: elaboración propia a partir de la base EC-PISAC.

Otro aspecto importante se relaciona con la bibliografía citada en los artículos. Un primer dato que resalta es que el $1,8 \%$ de ellos no cuenta con ninguna referencia bibliográfica, y un 3,5\% contiene entre 1 y 5 referencias. El 11,4\% no cita ningún otro artículo de revista y el $39,5 \%$ no cuenta con citas de trabajos publicados en inglés. El total de referencias bibliográficas, agrupadas en intervalos, puede observarse en la tabla 2:

Tabla 2. Cantidad de referencias bibliográficas

\begin{tabular}{|l|c|}
\hline \multicolumn{1}{|c|}{ Referencias bibliográficas } & \% \\
\hline Ninguna & 1,8 \\
\hline De 1 a 5 & 3,5 \\
\hline De 6 a 10 & 7,0 \\
\hline De 11 a 20 & 30,7 \\
\hline De 21 a 100 & 46,5 \\
\hline Más de 100 & 10,5 \\
\hline
\end{tabular}

Fuente: elaboración propia a partir de la base EC-PISAC. 
En cuanto a la antigüedad de la bibliografía, se observa que un 1,8\% de los artículos tiene como referencia más reciente a trabajos publicados antes de $1970 ; 0,9 \%$ a publicaciones de la década de $1980 ; 7,9 \%$ a textos de la década de 1990 y $89,5 \%$ a trabajos publicados después del año 2000. Por otra parte, no hay textos de los últimos 10 años en la bibliografía citada del $3,5 \%$ de los casos, mientras que un $17,5 \%$ de ellos cuenta con entre 1 y 5 referencias de esta antigüedad; $20,2 \%$ entre 6 y 10 ; $31,6 \%$ entre 11 y $20 ; 26,3 \%$ entre 21 y 50 , y $0,9 \%$ entre 51 y 100 . El peso relativo de la bibliografía de menos de 10 años de antigüedad en el total de la bibliografía citada es de hasta $10 \%$ (en $2,7 \%$ de los artículos); entre 10 y $25 \%$ (en $8,9 \%$ ), entre 25 y $50 \%$ (en $34,8 \%$ ), entre 50 y $75 \%$ (en $30,4 \%$ ) y más de $75 \%$ (en $19,6 \%$ de los artículos)

La cantidad de artículos de revistas referenciados, agrupados en intervalos, se consigna en la tabla 3 .

Tabla 3. Cantidad de referencias bibliográficas (artículos de revista)

\begin{tabular}{|l|c|}
\hline \multicolumn{1}{|c|}{ Referencias bibliográficas } & \% \\
\hline Ninguna & 11,4 \\
\hline De 1 a 5 & 41,2 \\
\hline De 6 a 10 & 29,8 \\
\hline De 11 a 20 & 12,3 \\
\hline De 21 a 100 & 5,3 \\
\hline
\end{tabular}

Fuente: elaboración propia a partir de la base EC-PISAC.

En términos relativos se constata que el peso de los artículos de revista citados, sobre el total de la bibliografía utilizada, fue de hasta $10 \%$ (en 9,8\% de los artículos); entre 10 y $25 \%$ (en $31,3 \%$ ), entre 25 y $50 \%$ (en $46,4 \%$ ), entre 50 y $75 \%$ (en $2,7 \%$ ). No se encontró asociación estadística entre la estrategia metodológica (cuantitativa, cualitativa o mixta) y el peso relativo que tienen los artículos de revista en el conjunto de la bibliografía.

Sobre el origen de los textos citados se verificaron los patrones que se observan en el Gráfico 2. El porcentaje de artículos que no cita ningún texto argentino ronda el 4\%, y es más bajo en relación con la ausencia de citas de origen estadounidense / europeo (cerca del 10\%) y latinoamericano (más de 27\%). En el caso de la bibliografía latinoamericana, el modo de la distribución se encuentra en el intervalo de 1 a 5 textos referenciados, siendo ésta la situación de casi el 50\% de los artículos. Para el caso de la bibliografía estadounidense / europea la distribución es prácticamente bimodal, con frecuencias relativas casi idénticas para los intervalos de 6 a 10 y de 11 a 20 textos citados. Tomados en conjunto ambos intervalos, ésta es la situación de aproximadamente el $40 \%$ de los artículos analizados. La bibliografía de origen argentino es la más citada: casi $40 \%$ de los artículos citan entre 11 y 20 textos de este origen.

Por otra parte, el peso relativo de la bibliografía de cada origen se puede observar en la siguiente tabla: 
Gráfico 2. Origen de la bibliografía.

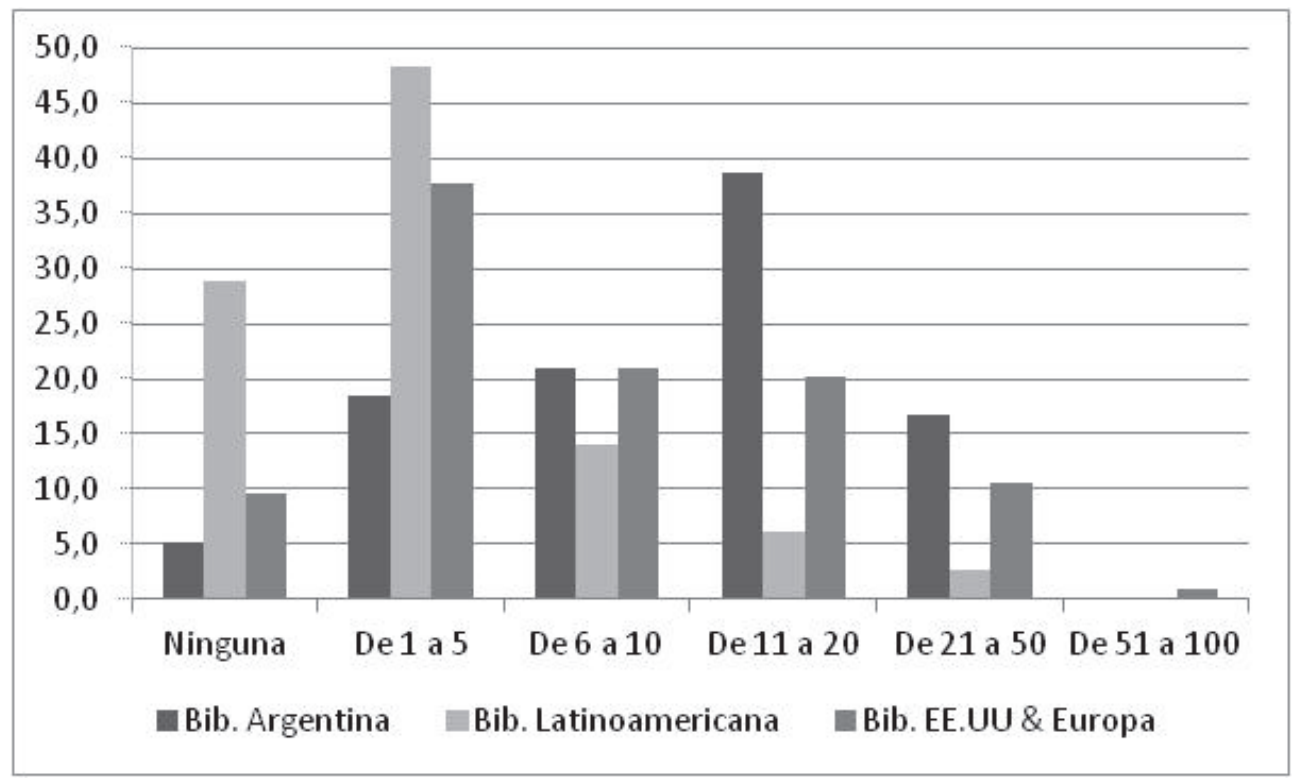

Fuente: elaboración propia a partir de la base EC-PISAC

Tabla 4. Peso relativo de la bibliografía argentina, latinoamericana, estadounidense y europea en el total de la bibliografía citada.

\begin{tabular}{|l|c|c|c|}
\cline { 2 - 4 } \multicolumn{1}{c|}{} & Bib. Argentina & Bib. Latinoamericana & Bib. EE.UU \& Europa \\
\hline No citados & 3,6 & 27,7 & 9,8 \\
\hline Hasta $10 \%$ & 2,7 & 25,0 & 11,6 \\
\hline De 10 a $25 \%$ & 8,9 & 23,2 & 25,9 \\
\hline De 25 a $50 \%$ & 34,8 & 17,9 & 25,0 \\
\hline De 50 a $75 \%$ & 30,4 & 4,5 & 19,6 \\
\hline Más de $75 \%$ & 19,6 & 1,8 & 8,0 \\
\hline
\end{tabular}

Fuente: elaboración propia a partir de la base EC-PISAC.

Como se ha señalado, el $38,4 \%$ de los artículos no tiene ninguna referencia a textos en inglés, pero entre aquellos que sí citan trabajos publicados en esta lengua, su peso relativo en el total de la bibliografía es de hasta $10 \%$ en el $20,5 \%$ de los casos; de 10 a $25 \%$ en el $15,2 \%$; de 25 a $50 \%$ en otro $15,2 \%$; de 50 a $75 \%$ en un $8,9 \%$ y de más de $75 \%$ en un $1,8 \%$ de los casos. A diferencia de lo registrado en relación con las citas de otros artículos de revista dentro de la bibliografía, en este caso sí se detectó una leve asociación estadística con la estrategia metodológica. En efecto, tendencialmente las citas de textos en inglés tienen mayor incidencia en las investi- 
gaciones cuantitativas o en aquellas basadas en métodos mixtos, en relación con las cualitativas.

Los artículos seleccionados fueron publicados en 81 revistas diferentes, en su amplia mayoría editadas en Argentina. En ningún caso se registran más de 4 artículos de una misma revista, y el promedio por revista es inferior a 2 publicaciones. El $77,2 \%$ de los artículos contó con evaluación por pares, y el 81,6\% fue incluido en revistas indizadas, con un abrumador predominio de Latindex: en efecto, cerca del $85 \%$ de ellas figura en esta base, aunque en muchos casos sólo constan en el Directorio. También se destaca el porcentaje de revistas incluidas en las bases de datos regionales SciELO (cerca del 35\%) y REDALyC (aproximadamente 25\%), así como en el Directory of Open Access Journals, DOAJ (23\%). La inclusión en las bases internacionales que nuclean a las revistas de corriente principal es excepcional: menos de $6 \%$ se encuentran en Scopus y cerca de $4 \%$ en WoS. Por otra parte, el 9,6\% de los artículos fue publicado en revistas impresas, mientras que el 31,6\% apareció en revistas digitales y el $58,8 \%$ restante en revistas que cuentan con versión impresa y digital. Del conjunto de artículos, el 72,8\% fue incluido en revistas que exigen resumen y palabras clave en un segundo idioma.

Finalmente, en relación con el impacto de estas publicaciones, se pudo establecer que el 76,3\% de ellas se encuentran registradas en Google Académico (GA), pero el nivel de citaciones es en general bajo: $13,8 \%$ de los casos que aparecen en GA no se encuentra citado en otras publicaciones; $57,5 \%$ está citado entre 1 y 5 veces; $8 \%$ entre 6 y 10 veces; $10,3 \%$ entre 11 y 20 veces; $5,7 \%$ entre 21 y 50 veces y $4,5 \%$ más de 50 veces.

\section{Discusión y conclusiones}

Una primera cuestión que quisimos destacar, a pesar de que se apuntaba a caracterizar con mayor profundidad los artículos basados en investigaciones empíricas, es que los investigadores argentinos le asignan importancia a una amplia variedad de tipos de publicaciones y de trabajos a la hora de seleccionar materiales que consideran relevantes para dar cuenta de los aportes locales de la investigación social. En cierto sentido, podría afirmarse que aunque el corpus contiene más de un $46 \%$ de artículos de revista, su exclusividad como forma de materialización de la producción académica está cuanto menos en disputa.

Este resultado es consistente con las muchas formas de resistencia que diversos actores individuales y colectivos, dentro del sistema de ciencias sociales argentino, vienen llevando a cabo desde hace años frente a las políticas científicas que apuntan a entronizar al paper como único producto científico legítimo, en desmedro de otros tipos de publicaciones, especialmente los libros. En este sentido puede recordarse, entre muchas otras iniciativas individuales y colectivas, la labor de la Comisión Interinstitucional de Elaboración de Criterios de Evaluación para las Humanidades y Ciencias Sociales (CIECEHCS), un grupo auto-gestionado de instituciones e investigadores que desde 2012 trabaja en la formulación de criterios específicos para la evaluación de la producción de las ciencias sociales y la humanidades, y que ha defendido la importancia central del libro y de las publicaciones en castellano.

No obstante, es posible que en esta cuestión estén en juego diferencias disciplinarias y generacionales. Baranger y Niño (e/p), por ejemplo, han mostrado que ciertas 
disciplinas de las ciencias sociales replican los modelos de producción de las ciencias naturales, con un claro predominio del paper en inglés, mientras que en otras el libro conserva un reconocimiento muy significativo. Beigel (2017b), por su parte, ha analizado la selección que los investigadores hacen de sus mejores publicaciones y ha notado que entre las generaciones más jóvenes hay mayor propensión a seleccionar artículos publicados en revistas mainstream. En este sentido, de las cinco publicaciones más destacadas de toda su carrera, los cientistas sociales menores de 45 años eligen en promedio 4,4 artículos de revista. Para Beigel esto es evidencia de que el paper se está volviendo cada vez más dominante en todos los campos científicos. Pero también debería considerarse que, independientemente de la posible mayor aceptación de los modelos internacionales de producción científica estándar por parte de los jóvenes, la publicación de libros, por sus características específicas, es más frecuente entre quienes acreditan carreras académicas más consolidadas, algo que está claramente correlacionado con la edad.

Más allá de esta variedad de publicaciones, también debe destacarse la legitimidad de diverso tipo de trabajos dentro de esta comunidad académica. Además de las investigaciones empíricas (más del 60\% del total del corpus) y teórico-conceptuales, y de las revisiones sistemáticas de literatura, llama la atención el peso asignado a los ensayos. ${ }^{6}$

Ahora bien, centrándonos específicamente en los artículos de revista, se pudo constatar que una mayoría de ellos, en línea con lo que se reporta en otros trabajos, es de carácter cualitativo (véase por ejemplo Piovani, 2015). El predominio de esta orientación metodológica en la investigación social argentina reciente es ampliamente conocido, y alcanza proporciones mucho más altas que la verificada en el corpus en el caso de las tesis doctorales (con la excepción de disciplinas tales como Economía y Demografía). En la medida en que, tal como señala el Writing Center de la Universidad de Wisconsin-Madison, ${ }^{7}$ el formato IMRAD no es muy utilizado en reportes que describen los resultados de estudios de caso o de campo, no debería sorprender que la difusión de este formato estándar sea bastante acotada en el conjunto de artículos analizado. Esta afirmación sobre la menor difusión del formato IMRAD en los reportes cualitativos está en línea con uno de los hallazgos, ya que se pudo determinar que en los artículos basados en investigaciones cuantitativas, al menos algunos de los componentes centrales de este formato -en particular la sección dedicada a métodos-, tienen una presencia mucho mayor. Por supuesto que estas consideraciones no implican un juicio sobre la calidad de los artículos, o de las investigaciones en las que se basan.

La adscripción disciplinar también contribuye a explicar la baja frecuencia de artículos del corpus que se ajustan al formato de escritura científico dominante. Tal como han mostrado Baranger y Niño (e/p), los estilos de publicación que denominan estándar y proto estándar, y que implican un apego a los modelos de las ciencias naturales, son mayoritarios en Argentina en disciplinas tales como la Antropología Biológica, la Economía y la Psicología (presumiblemente en sus variantes experimental y psicométrica), mientras que los trabajos analizados en este estudio se inscriben principalmente en la Sociología, la Antropología Social, la Comunicación Social y la Ciencia Política, disciplinas en las que, de acuerdo con la investigación

Para una definición de estos tipos de trabajo véase la nota 4.

https://writing.wisc.edu/ 
de los autores citados, predominan los estilos localistas y universalistas. El caso de la Ciencia Política argentina es bastante peculiar, dado que en muchos contextos científicos periféricos, en particular en América Latina, es fuerte la impronta estadounidense y, en consecuencia, la difusión del modelo estándar de paper.

Ya sea que se trate de reportes de estudios cuantitativos, cualitativos o triangulados, e independientemente de las consideraciones críticas que puedan hacerse en relación con el formato IMRAD, resulta llamativa la alta proporción de artículos en los que no se explicitan las decisiones metodológicas y/o no se sitúan los objetos de indagación en un campo problemático construido a partir de la revisión de otros trabajos temáticamente afines y recientes. Particularmente seria parece la alta proporción de trabajos que no dan cuenta de las decisiones de muestreo, aunque también resultó alta la de aquellos que no reportan las decisiones de producción y análisis de datos. Como se indicó en la sección de resultados, el reporte de las decisiones de muestreo es prácticamente nulo en los trabajos de corte cualitativo, a pesar de que la literatura especializada incluye habitualmente el tratamiento del tema "muestreo cualitativo".

Estos hallazgos son relevantes a la hora de pensar los procesos de formación en investigación y, más puntualmente, el lugar de la enseñanza de los métodos y técnicas de investigación social. Más allá de posibles déficits de la formación técnica en cuestiones puntuales como muestreo cualitativo, investigaciones previas han mostrado que, en general, la enseñanza de la metodología tiende a desentenderse del problema de la escritura científica, centrándose casi exclusivamente en las discusiones epistemológicas, en los debates metodológicos, en el diseño de la investigación y en la producción y análisis de datos (Piovani, xxxx). Un mayor énfasis en la revisión de los formatos habitualmente utilizados a nivel internacional para reportar los resultados de la investigación, durante los procesos formativos, podría redundar en la producción de textos científicos más acordes con los estándares científicos vigentes que, entre otras cosas, requieren la explicitación de las decisiones metodológicas.

La estructura de la bibliografía también permite proponer algunas lecturas relevantes. En primer lugar, se destaca la baja presencia relativa de artículos de revista y de textos en inglés en la bibliografía citada. Como hipótesis, podría argumentarse que esto resulta consistente con la escasa inclusión de revisiones de literatura / estados de la cuestión en los artículos analizados, en la medida que ellos se construyen -habitualmente- sobre la base de literatura reciente y de frontera, que suele publicarse en la forma de papers y que en general no se traduce. Esta hipótesis encuentra sostén adicional en las estadísticas oficiales de descarga de artículos desde la biblioteca electrónica de ciencia y tecnología del MINCYT, que provee acceso a las bases de datos internacionales a todas las instituciones públicas de investigación y de educación superior. En el período comprendido entre enero de 2008 y diciembre de 2017, los investigadores de ciencias sociales descargaron 2.263.192 artículos, frente a los más de 30 millones descargados por sus colegas de ciencias exactas y naturales, y la descarga promedio por investigador por año fue de 141 en estas disciplinas, en comparación con 12 en las ciencias sociales ${ }^{8}$.

Sobre el origen de la bibliografía se destaca el alto peso de la producción local, pero no siempre en formato de artículos de revista que, como se ha señalado, tienen una presencia relativamente baja en el total de citas y referencias. La bibliografía

8 http://www.biblioteca.mincyt.gob.ar/estadisticas/disciplinas 
latinoamericana aparece poco referenciada, a pesar de su importancia obvia dadas las similitudes que presentan muchos procesos socio-históricos dentro de la región. Más allá de la retórica latinoamericanista en la comunidad de ciencias sociales, la presencia mucho mayor de literatura estadounidense y europea evidencia la importancia que los grandes centros mundiales de producción de conocimiento tienen en los ámbitos científicos periféricos, especialmente en relación con la literatura teórica y metodológica. La poca proporción de textos en inglés y en otros idiomas (francés, alemán, italiano, etc.), y de artículos de revista, sugiere que los textos de origen estadounidense y europeo que se citan son en general libros que ya han sido traducidos. A pesar de todo lo expuesto, resalta positivamente que la mayor parte de la bibliografía citada es, en general, bastante reciente.

Los resultados sobre los soportes de publicación de los artículos analizados están en línea con lo reportado en estudios previos (por ejemplo Beigel, 2017b) que muestran la existencia de un circuito local de publicaciones periódicas, compuesto por revistas no indizadas $-\mathrm{O}$ al menos no indizadas en las bases internacionales de corriente principal- en las que publican investigadores poco apegados a los estándares internacionales del Sistema Académico Mundial, y que suelen ser generalmente reconocidas para la consolidación de carreras docentes en universidades no metropolitanas. En el caso argentino, un relevamiento llevado a cabo en el marco del PISAC mostró la existencia de más de 450 revistas locales de ciencias sociales y humanas activas, la mayoría de ellas con limitada circulación y visibilidad, y niveles poco profesionalizados de gestión editorial (Piovani, 2015). La mayoría de las revistas en las que fueron publicados los artículos incluidos en el corpus pertenecen, en efecto, a este circuito local. Por otra parte, cabe señalar que los artículos de estas revistas tienen, en general, un bajo nivel de citación, y esto es consistente con los hallazgos de este trabajo en relación con el impacto de los artículos incluidos en el corpus.

\section{Referencias}

Alatas, S. F. (2003), "Academic Dependency and the Global Division of Labour in the Social Sciences." Current Sociology 51, 6: 599-613.

Baranger, D. y Niño, F. (e/p), "El espacio de las disciplinas sociales en el CONICET". En: Baranger, D.; Beigel, F. y Piovani, J. I. (eds.), Las ciencias sociales en la Argentina contemporánea. Buenos Aires: Clacso (en preparación).

Beigel, F. (2017a), “Científicos Periféricos, entre Ariel y Calibán. Saberes Institucionales y Circuitos de Consagración en Argentina: Las Publicaciones de los Investigadores del CONICET". Dados-Revista de Ciências Sociais 60, 3: 825 a 865.

Beigel, F. (2017b), “Un mapeo de las ciencias sociales argentinas". Dialogo Global 7, 4:3133-36.

Beigel, F. y Salatino, M. (2015), “Circuitos segmentados de consagración académica: las revistas de Ciencias Sociales y Humanas en la Argentina”. Información, cultura y sociedad 32: 11-35.

Brain. W. R. (1965), "Structure of the Scientific Paper”. British Medical Journal 2: 868-869

Burawoy, M. (2005), "For Public Sociology". American Sociological Review 70:4-28.

Day, R. A. (1989), "The Origins of the Scientific Paper: The IMRAD Format". American Medical Writers Association Journal 4, 2: 16-25. 
Hanafi, S. (2011), "University systems in the Arab East: Publish globally and perish locally vs. publish locally and perish globally". Current Sociology 59, 3: 291-309.

Ortiz, R. 2009. La Supremacía del inglés en las ciencias sociales. Buenos Aires: Siglo XXI.

Peirano, F.; Freibrun, N. y Sleiman, C. (2015), "Las nuevas políticas públicas y el cambio en la representación social de los doctores". Sociedad 34: 5-11

Piovani, J. I. (2015a), "El Programa de Investigación sobre la Sociedad Argentina Contemporánea". Sociedad 34: 85-105.

Piovani, J. I. (2015b), “Algunos desafíos para la evaluación académica en Ciencias Sociales.” Controversias y Concurrencias Latinoamericanas 7, 12: 25-40.

Piovani, J. I. (2017), “Argentina bajo investigación”. Dialogo Global 7, 4:31-33.

Sollaci. L. B. y Pereira. M. G. (2004). "The introduction, methods, results, and discussion (IMRAD) structure: a fifty-year survey". Journal of the Medical Library Association 92, 3: 364-371.

Unzué, M. (2015), “Nuevas políticas públicas de formación de doctores en Argentina”. Sociedad 34: 12-34. 
